\title{
The Liverpool Ortho-Plastic Model
}

\author{
N Sargazi, A Mostafa and A El-Gawad* \\ Mersey Regional Plastic Surgery Unit, Whiston Hospital, UK \\ Department of Trauma and Orthopaedics, Royal Liverpool and Broad green University Hospitals, UK
}

Submission: November 22, 2016; Published: December 06, 2016

*Corresponding author: Nastaran Sargazi, Department of Burns and Plastic Surgery, Whiston Hospital, UK. jaandres@ono.com

\begin{abstract}
Introduction: The ever increasing incidence of trauma and its associated multiple complex injuries has paved the way for an ortho-plastic approach to the management of such patients within the recently introduced "Major Trauma Centres" (MTCs). Unfortunately, many MTCs lack the presence of an on-site plastic surgical team, which may delay treatment in those requiring input for soft tissue coverage. The presence of plastic surgeons within a MTC or regional trauma units does not only improve the level of care provided in acute injuries but enables the establishment of multiple elective services for those with chronic bone and joint conditions.
\end{abstract}

Patients and Materials: In this paper we describe the impact on the trauma and elective services provided by our regional trauma and orthopaedic unit at The Royal Liverpool University Hospital following the appointment of a full-time on-site consultant plastic surgeon.

Results: The addition of a consultant plastic surgeon within our busy regional trauma and orthopaedic (T\&0) unit and has resulted in a profound reduction in time to review and definitive soft tissue coverage in trauma patients in addition to a substantial reduction durations and cost of in-patient stay. Furthermore, we have been able to show a similar trend in our elective sarcoma and foot and ankle patients.

Discussion: The constant availability of a plastic surgeon within out T\&O unit has facilitated the creation of multiple new services including the foot and ankle MDT to enable a multidisciplinary approach to the management of such patients.

Conclusion: This ortho-plastic model is one which should be considered and replicated in all large regional T\&0 units or MTCs.

Keywords: Ortho-plastic, Trauma, Sarcoma, Multidisciplinary, Foot and ankle

\section{Introduction}

The incidence of trauma continues to increase, resulting in an exponential rise in mortality and morbidity globally [1]. Patients commonly present with multiple injuries, hence accounting for trauma being the sixth most common leading cause of death worldwide [1]. In England alone, there are an estimated 20,000 cases of major trauma per annum, resulting in approximately 5,400 deaths annually, with the remaining cohort experiencing various long term disabilities as a result of their injuries [2].

The immediate management of such patients has been quoted to cost between 300-400 million pounds per year [2]. Such costs are predominantly related to patients presenting with a range of complex injuries, requiring input from specialities which is usually not available within the same unit, hence resulting in costly patient transfers between centres and leading to delays in treatment [3].

Such deficiencies in the care of those with severe complex injuries, was first highlighted by the Royal College of Surgeons in 1988 and accounts for an estimated 20 per cent increase in the rates of in-hospital mortality in such patients compared to other countries including USA [2], leading to 3,000 preventable trauma-related deaths a year in England and Wales alone [4]. Following multiple reviews of trauma care, the concept of "Regional Trauma Networks" (RTNs) was introduced within the UK, leading to a substantial changes in the management of this cohort [4].

RTNs consist of specialised trauma centres, or "Major Trauma Centres" (MTCs), of which there are currently 26 centres throughout the UK, all aimed at delivering organised regional trauma care for patients with complex injuries through availability of a range of specialities within one unit [5]. In addition to MTCs, RTNs also consists of "trauma units" who have facilities to provide initial stabilisation of injuries, and where possible, the definitive management of such patients [6]. "The Liverpool Collaborative MTC", consists of the collaboration of three units; Aintree Univeristy Hospital, The Walton Centre 


\section{Orthopedics and Rheumatology Open Access Journal}

and the Royal Liverpool University Hospital [7]. Whilst Aintree University Hospital acts as the primary MTC in this region, The Royal Liverpool University (RLH), is a large unit with a range of specialities within a single unit and also receives a larger portion of patients.

Given the increasing number of trauma patients admitted to RLH, expansion of our trauma and orthopaedic department, a regional unit which also consists of ortho-plastic, sarcoma and foot and ankle services has resulted in a surprisingly large improvement in the care of both those with acute and chronic injuries. In this paper we describe the progress of our services since our original paper, "A full time regional ortho-plastic unit; initial results" [8].

\section{Patients and Materials}

In this paper we will describe the changes in the services provided by our regional trauma and orthopaedic unit at The Royal Liverpool University Hospital, in terms of both acute trauma and elective care, following the appointment of a fulltime on-site consultant plastic surgeon within our department. In particular, we focus on the establishment of new elective services following the addition of this speciality within our orthopaedic unit.

\section{Results}

In our original paper, "A full time regional ortho-plastic unit; initial results" [8], we described our experience of trauma care, focusing on the management of trauma patients with injuries requiring input from both orthopaedic and plastic surgeons [8]. This combined ortho-plastic approach is a well established concept in the management of those with extremity trauma, with its cornerstone being combined surgical care from both specialities [9]. This has been emphasised by both the British Orthopaedic Association (BOA) and British Association of Plastic and Reconstructive Surgeons (BAPRAS) who have provided recommendations for the management of such patients, in particular those with open fractures of the lower limb [10]. Not only can such recommendations be applied to all open fractures but also other disease processes involving the extremities where input from both these specialities is required.

These recommendations have recently been published as national guidelines by NICE [11], who recommend initial debridement within 24 hours of injury by both orthopaedic and plastic surgeons with definitive soft tissue coverage not exceeding 72 hours [11]. In current practice, many MTCs, including our regional MTC, Aintree Univeristy Hospital, do not currently have capacity for an on-site plastic surgeon, hence deficiencies remain in management of trauma patients, in particular those with complex open fractures [9].

Our regional trauma unit at RLH receives a large number of complex cases including both open and complex closed fractures in addition to patients with chronic complicated bone and joint diseases. The vast majority of these patients require input from both the experienced orthopaedic surgeons within our unit in additional to our plastic surgery colleagues from the regional plastics unit, Whiston hospital.

Whilst this joint input is extremely valuable, we found that the lack of a designated plastic surgeon working within our team and the logistics involved with referring and transferring patients to the regional plastics unit for soft-tissue input resulted in unnecessary delays in management and prolonged in-patient stay in such a cohort. Therefore, a full-time consultant plastic surgeon was appointed within our trauma and orthopaedic (T\&0) unit in September 2014. The blending of the two specialities enabled simultaneous application of the principles and practices of both specialities to clinical problems, hence provided a more comprehensive and efficient management process for such patient. This ortho-plastic model was not only applied to our trauma cases but also to patients with malignant and infected disease processes involving both the lower and upper extremities, resulting in the establishment of new elective services within our regional T\&0 unit which we will describe in greater detail in this paper.

\section{Discussion}

\section{A.Ortho-plastic model of trauma care}

As highlighted above, management of trauma, in particular severe complex open fractures requires a combined orthoplastic approach to improve rates of limb salvage and functional outcome in patients in addition to reducing the incidence of deep infections and non-unions [12]. Prior to September 2014, all trauma patients admitted to our regional T\&O unit, underwent initial skeletal stabilisation under the care of our orthopaedic surgeons. Whilst this timely debridement and bony fixation was undertaken at our unit, we found that the lack of an on-site plastic surgeon and the need for referral of patients to our colleagues at a the regional plastic surgical unit resulted in delays in soft tissue coverage, with many inpatients having to wait for long periods of time within our T\&O unit whilst arrangements were made for a plastic surgeon to visit our unit or for the patient to be transferred to the regional plastic surgery unit (retrospective group).

Following the addition of a consultant plastic surgeon within our unit in September 2014, all trauma patients requiring plastic surgical input for soft tissue coverage were managed within our regional T\&O unit (prospective group), unless a free flap was required, in which case they were transferred to the regional plastic unit due to current lack of facilities for free flap construction and monitoring at our T\&O unit [8]. The initial results of such intervention was examined and published in our original paper [8], showing a $83 \%$ decrease in time until plastic surgical review and $67 \%$ decrease in time until soft tissue coverage in addition to a $79 \%$ and $78 \%$ reduction in length and cost of inpatient stay respectively [8] (Table 1). 
Table 1: Overview of trauma care.

\begin{tabular}{|c|c|c|c|}
\hline & $\begin{array}{c}\text { Time to } \\
\text { Review } \\
\text { by Plastic } \\
\text { Surgeons } \\
\text { (Median) }\end{array}$ & $\begin{array}{c}\text { Time to } \\
\text { Definitive } \\
\text { Soft Tissue } \\
\text { Coverage } \\
\text { (Median) }\end{array}$ & $\begin{array}{c}\text { Cost of } \\
\text { Inpatient Stay } \\
\text { (Average) }\end{array}$ \\
\hline $\begin{array}{c}\text { Retrospective } \\
\text { Group }\end{array}$ & 6 days & 9 days & $£ 12,452.66$ \\
\hline $\begin{array}{c}\text { Prospective } \\
\text { Group }\end{array}$ & 24 hrs & 3 days & $£ 2750.28$ \\
\hline
\end{tabular}

As previously mentioned, these vast improvements were not only due to constant availability of a plastic surgeon within our unit but also due to the increased ease of communication between the orthopaedic and plastic surgeons in addition to the presence of a dedicated trauma theatre available for the management of such trauma patients [8]. This ortho-plastic model has highlighted the positive impact of combined surgical care in enabling management of bony and soft tissues injuries, hence allowing earlier mobilisation and discharge of patients to help improve functional outcomes and reduce complications associated with prolonged lengths of in-patient stay [8]. Hence we feel it is a model which should be used in other regional MTCs.

The constant availability of a plastic surgeon within the orthopaedic team did not only improve the care of trauma patients but also encouraged referral from other subspecialties within T\&O, including the foot and ankle and sarcoma teams, hence leading to the establishment of new services within these fields which we will describe in detail below.

\section{B.Ortho-plastic model of sarcoma care}

Musculoskeletal tumours are relatively rare, in particular soft tissue sarcomas, which have an estimated incidence of 3,800 per year and account for approximately $1 \%$ of all cancers [13]. Such lesions arise from mesenchymal tissue and are of varying histological subtypes [14], with the majority being locally invasive and metastasising haematogenously or occasionally via the lymphatic system [15].

Due to its rarity, sarcoma care is a niche area within the field of trauma and orthopaedics, and requires specialist input for diagnosis and management, thereby necessitating input from a range of healthcare professionals [14]. The multidisciplinary care of extremity sarcomas is carried out within tertiary centres and consists of input from numerous specialities, including oncologists, pathologists, radiologists and plastic and orthopaedic surgeons to provide comprehensive treatment of such patients [14].

The gold standard management of extremity soft tissue sarcomas is surgical resection [15], with wide tumour resection and limb-salvage being the main goals of such interventions, which has resulted in a decline in amputation rates in this patient cohort [14]. In reality, operative management of extremity sarcomas are frequently complex, this in addition to the increasingly incidence of unplanned primary excisions which require wide re-excision, require the collaboration of both orthopaedic and plastic surgeons within a single unit [15], to ensure timely and satisfactory skeletal stabilisation and optimal soft tissue coverage [16].

There are currently ten tertiary centres for management of soft tissue sarcomas in England [17], however, like MTCs, many rarely have a designated on-site plastic surgeon working within their unit. Given the rarity of such a condition and the resultant unique presentation of each lesion between different patients, detailed review of imaging and discussion of management options by both specialities prior to entering the operating room is of great importance to optimise both management of the disease process and functional outcome in patients [15]. The lack of an in-team plastic surgeon within such units may result in delays in interventions relating to the logistics of attempting to arrange combined surgical intervention within a single unit, hence have an impact on patient outcomes [15].

The local sarcoma service for Merseyside and Cheshire, "The Liverpool Sarcoma Service" is based within our regional T\&O unit at RLH. This multi professional group serves approximately 2.3 million people within the Merseyside and Cheshire region in addition to providing diagnostic and treatment services to roughly 1.6 million patients based in the South Cumbria and Lancaster region. The service receives an average of 20-25 new referrals each week, most of which are obtained from primary care providers via the nationwide "urgent two week referral rule" for suspected cancers, with 120 new cases being diagnosed annually. Once received, patients are discussed in the MDT meetings within the same week of referral and outcome is faxed to the referring team.

Since the appointment of the on-site consultant plastic surgeon within our regional trauma unit, there has been a profound improvement in the ease of communication and collaboration between the two specialities in the management of soft tissue sarcomas. Furthermore, the addition of a plastic surgeons within our unit has resulted in the presence and involvement of this specialist in the weekly sarcoma MDTs, hence providing a truly multidisciplinary approach to the preoperative planning of patients. The MDTs occur twice weekly on Mondays and Fridays, with the Monday meetings attended by the sarcoma surgeon, radiologist and the sarcoma specialist nurses and aimed at discussing new patients, roughly 20-30 in number. Approximately 20-25 patients are discussed in the regional MDT which takes place on Fridays and is attended by all members of the MDT previously mentioned, in addition to clinical and medical oncologists, histopathologists and most recently our onsite plastic surgeon, a member who was not previously present within the MDT meetings, hence resulted in additional meeting being arranged by the sarcoma surgeon to obtain input from this speciality regarding soft tissue coverage and led to delays in arranging for both specialities to be present in theatre at the time of tumour resection. 
Given the efficiency of the system and the resources available, there is currently no waiting time for surgery with those with high grade sarcomas being operated on within a few days of diagnosis. It is important to highlight the importance of a truly holistic approach to this cohort of patients, which is provided both pre- and post operatively by our team of specialist sarcoma and Macmillan nurses, psychologists, social workers in addition to physio and occupational therapists. Furthermore, the patients are invited to join a 2 monthly support group and are clinically followed up for an average of 10 years in routine cases.

\section{C.Ortho-plastic model in foot and ankle disease}

Foot and ankle surgeons encounter a range of disease processes, from acute trauma to chronic conditions including arthritis and diabetes-related foot disease to congenital and acquired deformities [18]. Regardless of aetiology, substantial physical limitations can occur in a large proportion of these patients, hence impacting on long-term functional outcome [18].

As with other areas, the ortho-plastic model of foot and ankle reconstruction aims to maximise limb function whilst repairing or replacing damaged or absent soft tissue, and often pertains to infections, traumatic injuries and congenital or acquired foot and ankle deformities [19]. In order to achieve such goals, a multidisciplinary approach involving the input of plastic surgeons is required to optimise outcomes of successful limb salvaging methods and to address soft tissue defects before they progress to a state of non-viability and result in avoidable amputations, which will no doubt have a longstanding physical and psychological impact on such patients [19].

Globally diabetes mellitus has become the fastest progressing chronic disease, affecting 300 million people worldwide [20]. In England alone, 3.1 million adults are estimated to be affected with this condition, which is set to rise to 4.6 million by 2030 [21]. Its associated long-term complications, in particular the resultant neuropathy and associated poor circulation observed in many predisposes patients to bone and soft tissue deformities, ulcers and subsequent infections, leading to approximately 6,000 amputations per year in England [21]. This alongside other associated complications pose a heavy economical burden on an already stretched National Health Service, with the cost of diabetic care reaching almost $£ 10$ million in 2011 [21].

In order to minimise such impact on healthcare systems, there has been a great emphasis on disease prevention and progression in those already diagnosed by close monitoring of diabetic control and more importantly, patient empowerment [20]. In relation to diabetic foot disease, there has been a great deal of patient education regarding foot self-care behaviours including daily foot inspections, adequate foot hygiene and appropriate footwear, in addition to frequent limb screening by trained professionals in order to identify "at risk" foots and manage them accordingly to prevent progression of disease [20].
Prevention of diabetic-related foot disease involves active involvement of healthcare professionals but more importantly engagement and self motivation of patients [20]. Where one element is lacking, complication unfortunately arise resulting in a large proportion of these patients developing complications which requires surgical input [19]. Whilst soft tissue damage is readily evident in many, a substantial proportion of these patients also develop underlying osseous damage, which can result in pathological fractures, non-unions, segmental bony loss, osteomyelitis and subsequent sepsis [19]. Debridement of such wounds aims to discard all contaminated, non-viable and necrotic tissue, to provide a healthy bed of soft tissue and bone to allow satisfactory limb reconstruction and avoid early amputations [19].

Both debridement, which may be relatively extensive, and subsequent reconstruction which can be complex requires joint input from orthopaedic and plastic surgeons to produce a stable osseous construct around which soft tissue coverage can be created [19]. Soft tissue reconstruction varies depending on the extent of tissue loss, and can range from closure via secondary or delayed primary closure all the way to skin grafts and local or free flaps where large defects are present [19].

As highlighted above, the large proportion of foot and ankle patients present with chronic disease processes which not only involve bone but have a proportional impact on surrounding soft tissue [19]. Such cases are usually complex and involve numerous surgical interventions to optimise healing, hence emphasise the importance of a combined ortho-plastic model for management of such patients [19]. Unfortunately, as with trauma and sarcoma patients, this combined approach is usually not available at most centres, which like the patient cohorts above may cause delays to surgical management and subsequent healing of such patients.

Certainly, prior to September 2014, this was a frequently encountered problem in the our foot and ankle team within our regional T\&O unit, which receives approximately 30 new referrals per month, although this can vary. Following the addition of our on-site consultant plastic surgeon, we have been able to expedite the management of these patients both in an acute and elective setting by preventing delays relating to providing both specialities within the same operating room at the same period in time. Furthermore, the presence of our plastic surgeon has led to the creation of a foot and ankle MDT. The MDT meets monthly and consists of the orthopaedic and plastic surgeons, radiologists, foot and ankle specialist nurse and podiatrists. In addition to providing a platform for discussing complex cases, these meetings serve as a teaching opportunity for junior trainees regarding the management of such cases.

\section{Conclusion}

Our follow up study has highlighted the advantages of providing an ortho-plastic approach to the management of common orthopaedic conditions. This has been created by the 
addition of a single plastic surgeon within our busy regional trauma and orthopaedic unit and has had a profound impact on not only the care of our acute trauma patients but has also provided the foundations and resources for creation of multiple elective services.

This is a model which has not only improved patient care but providing a multidisciplinary approach to managing complex bone and joint disease but also reduced delays in obtaining such input from colleagues based outside our unit. The reduction in delays by enabling joint input by both orthopaedic and plastic surgeons within a single unit has also helped to reduce costs associated with patient transfer to regional units for soft tissue coverage and prolonged inpatient stay relating to delays in treatment.

Furthermore, the ability to work in side-by-side within a single unit has led to the referral and acceptance of a larger number of complex cases by our various teams. This increased frequency of exposure to such cases is no doubt of great educational benefit to both orthopaedic and plastic trainees and provides the foundations for more research within our unit. Hence we believe that this ortho-plastic model is one which should be considered in all large regional T\&O units or MTCs.

\section{Acknowledgement}

We would like to thank Sarah Massey from the Liverpool sarcoma service and Christine Atkinson from the Liverpool foot and ankle team for their help and input with the contents of this paper.

\section{References}

1. Alberdi F, Garcia I, Zabarte M (2014) Epidemiology of trauma. Med Internsiva 38(9): 580-588.

2. Major trauma care in England -national audit office 2010.

3. Wui LW, Shaun GE, Ramalingam G, Wai KM (2014) Epidemiology of trauma in an acute care hospita in Singapore. J Emerg trauma Shock $7(3): 174-179$.

4. Davenporarmy t RA, Tai N, West A, Bouamra O, Aylwin C, Woodford $\mathrm{M}$, et al. (2010) A major trauma centre is ar specialty hospital not a hospital of specialties. Br J Surg 97(1): 109-117.

5. Jansenj JO, Morison JJ, Tai NR, Midwinter MJ (2015) A survey of major trauma centre staffing in England. J R Army Med Corps 161(4): 341344.
6. Metcalfe D, Perry DC, Bouamra O, Salim A, Woodford M, et al. (2016) Regionalisation of trauma care in England. Bone Joint J 98(9): 12531261.

7. Map of major trauma centres (2012).

8. Sargazi N, El-Gawad A, Narayan B, Bell D, Shanks L, Nayagam S, et al. (2016) A full time regional ortho-plastic unit; Initial Results. J Plas Reconstructive Aesthet Surg 69(4): 572-573.

9. Wright TC, Chummun S, Chapman TWL, Khan U (2015) Role of plastic surgeons in major trauma centres in UK - is there a need for plastic surgery intervention in patients admitted to MTCs? The Bulletin 97(10): 445-447.

10. Boast 4 Guidelines (2016) Management of severe open fractures.

11. NICE (2016) Fractures (complex): assessment and management. NICE guideline 37.

12. Wordsworth M, Lawton G, Nathwani D, Pearse M, Naique S, Dodds A et al. (2016) improving the care of patients with severe open fractures of the tibia. Bone Joint J 98(3): 420-424.

13. Sarcoma (2015) the bone and soft tissue cancer charity.

14. Papagelopoulos PJ, Mavrogenis AF, Mastorakos DP, Patapis P, Soucacos PN (2008) Current concepts for management of soft tissue sarcomas of the extremities. J Surg Orthop Adv 17(3): 204-215.

15. Nystrom LM, Reimer NB, Reith JD, Dang L, Zlotecki RA, Scarborough MT, et al. (2013) Multidisciplinary management of soft tissue sarcomas. Scientific World Journal 28: 853462.

16. Siegel GW, Biermann JS, Chugh R, Jacobson JA, Lucas D, Feng M, et al. (2015) The multidisciplinary management of bone and soft tissue sarcoma: an essential organizational framework. J Multidiscip Healthc 19(8): 109-115.

17. (2016) Sarcoma specialist centres - Sarcoma UK.

18. Hunt KJ, Alexander I, Baumhauer J, Brodsky J, Chiodo C, Daniels T, et al. (2014) The orthopaedic foot and ankle outcomes research (OFAR) network: feasibility of a multicenternetwork for patient outcomes assessment in foot and ankle. Foot Ankle Int 35(9): 847-854.

19. Weistein RB, Larsen A (2004) Rotational Myoplasty for the treatment of osteomyelitis in foot and ankle. In: Ruch JA et al. (Eds.), Reconstructive surgery of the foot and leg: Update 2004. The Podiatry Publishing Co, Tucker, Georgia.

20. Bonner T, Foster M, Spears-Lanoix E. Type 2 diabetes_related foot care knowledge and foot self-care practice interventions in the United States: a systematic review of the literature. Diabet Foot Ankle 17(7): 29758.

21. McInnes AD (2012) Diabetic foot disease in the United Kingdom. J Foot Ankle Res 5(1): 26.

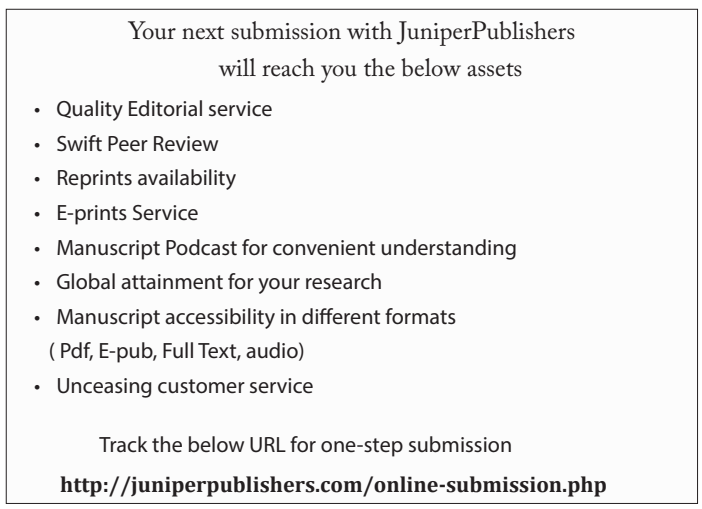

